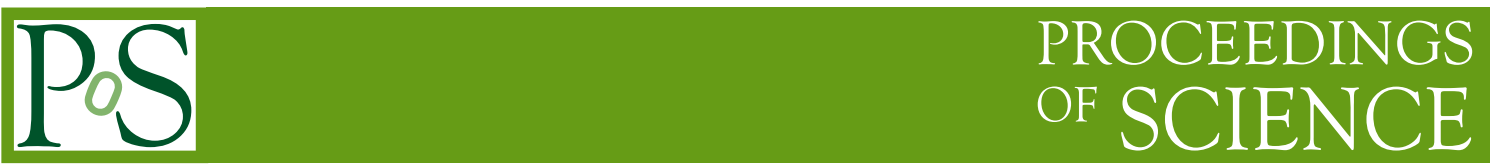

\title{
CMS MTD Barrel Timing Layer: Precision Timing at the HL-LHC
}

\author{
Badder Marzocchi ${ }^{a, 1, *}$ \\ ${ }^{a}$ Northeastern University, Boston (US) \\ E-mail: badder.marzocchi@cern.ch
}

The Compact Muon Solenoid (CMS) detector at the CERN Large Hadron Collider (LHC) is undergoing an extensive Phase II upgrade program to prepare for the challenging conditions of the High-Luminosity LHC (HL-LHC). A new timing detector in CMS will measure minimum ionizing particles (MIPs) with a time resolution of 30-40 ps for MIP signals at a rate of $2.5 \mathrm{Mhit} / \mathrm{s}$ per channel at the beginning of HL-LHC operation. The precision time information from this MIP Timing Detector (MTD) will reduce the effects of the high levels of pileup expected at the HL-LHC, bringing new capabilities to the CMS detector. The barrel timing layer (BTL) of the MTD will use sensors that are based on LYSO:Ce scintillation crystals coupled to SiPMs with TOFHIR ASICs for the front-end readout. In this talk we will present motivations for precision timing at the HL-LHC and an overview of the MTD BTL design, including ongoing R\&D studies targeting enhanced timing performance and radiation tolerance.

\footnotetext{
*** The European Physical Society Conference on High Energy Physics (EPS-HEP2021), ***

*** 26-30 July $2021 * * *$

*** Online conference, jointly organized by Universität Hamburg and the research center DESY ***

${ }^{1}$ On behalf of the CMS collaboration

* Speaker
} 


\section{Introduction}

For the HL-LHC phase a nominal instantaneous luminosity of $5.0 \times 10^{34} \mathrm{~cm}^{-2} \mathrm{~s}^{-1}$ with an average pile up of 140 is expected, while also an ultimate scenario with an instantaneous luminosity of $7.5 \times 10^{34} \mathrm{~cm}^{-2} \mathrm{~s}^{-1}$ and an average pileup of 200 will be delivered. The high collision rate during the LHC operation will result in radiation damage that will degrade the performance of the CMS subdetectors. In addition, the spatial overlap of tracks and energy deposits from the pile up interactions will have a negative impact on the identification and reconstruction of the physics objects produced in the hard interactions.

Because of this, The CMS [1] collaboration has approved the inclusion of the MIP Timing Detector (MTD)[2] in the upgrade plan for the HL-LHC era. The MTD detector will give timing information for MIPs with 30-40 ps resolution at the beginning of HL-LHC operation, degrading slowly as a result of radiation damage to $50-60 \mathrm{ps}$ by the end of HL-LHC operations. This subdetector will allow to perform a $4 \mathrm{D}$ vertex reconstruction, exploiting both the position and timing of the tracks and improving the performance of the vertex reconstruction. In particular, Figure 1 (left) shows that many of the vertices that appear to be merged in the spatial dimension are clearly separated when time information is available. In addition, Figure 1 (right) shows the reduction of the number of pileup tracks incorrectly associated with the hard interaction vertex for different MTD timing resolution hypotheses. The line density for a 200 pileup scenario is expected to reach values up to $1.8 \mathrm{~mm}^{-1}$. Assuming an MTD time resolution of $30-40 \mathrm{ps}$ this value can be reduced back to LHC-Run2 levels of about $0.8 \mathrm{~mm}^{-1}$.

The reconstruction and identification of other physics objects such as b-jets, photons, lepton isolation or the transverse missing momentum will also improve thanks to the addition of the timing information. These improvements in the objects will impact on flagship analyses such as the $\mathrm{HH}$ search, where about $15 \%$ increase in sensitivity is expected. At the same time, the timing information will bring unique opportunities for long-lived particle searches, where the time delay of the particles can be used to discriminate signal from background. In addition, the MTD detector will allow to perform a particle identification based on the Time-Of-Flight (TOF) measurement of charged particles, allowing to distinguish between pions, kaons and protons. This information will have a very strong impact on the reach of Heavy Ion and Flavour Physics analyses.

Following the layout of the CMS detector and the need for the MTD to fit into the detector, the MTD must be divided into two sections, the Barrel Timing Layer (BTL), covering $|\eta|<1.48$, and the Endcap Timing Layer (ETL), covering $1.6<|\eta|<3.0$. The MTD is required to last throughout the full HL-LHC era, reaching an integrated luminosity of about $3000 \mathrm{fb}^{-1}$. Both subdetectors will experience differing radiation dose through their lifetime: $1.65 \times 10^{14}-1.90 \times 10^{14} \mathrm{neq} / \mathrm{cm}^{2}$ for BTL and $1.50 \times 10^{15}-1.60 \times 10^{15} \mathrm{neq} / \mathrm{cm}^{2}$ for ETL, using FLUKA simulation. Because of this, different technologies have been chosen for designing the two subdetectors, in particular LYSO:Ce crystal bars coupled to SiPM readout for BTL, and LGAD sensors for ETL.

\section{BTL detector design}

The Barrel Timing Layer is a thin, cylindrical detector that will be housed inside the Tracker Support Tube (TST). The inner boundary is located at a radius of $1148 \mathrm{~mm}$ and extends up to a 

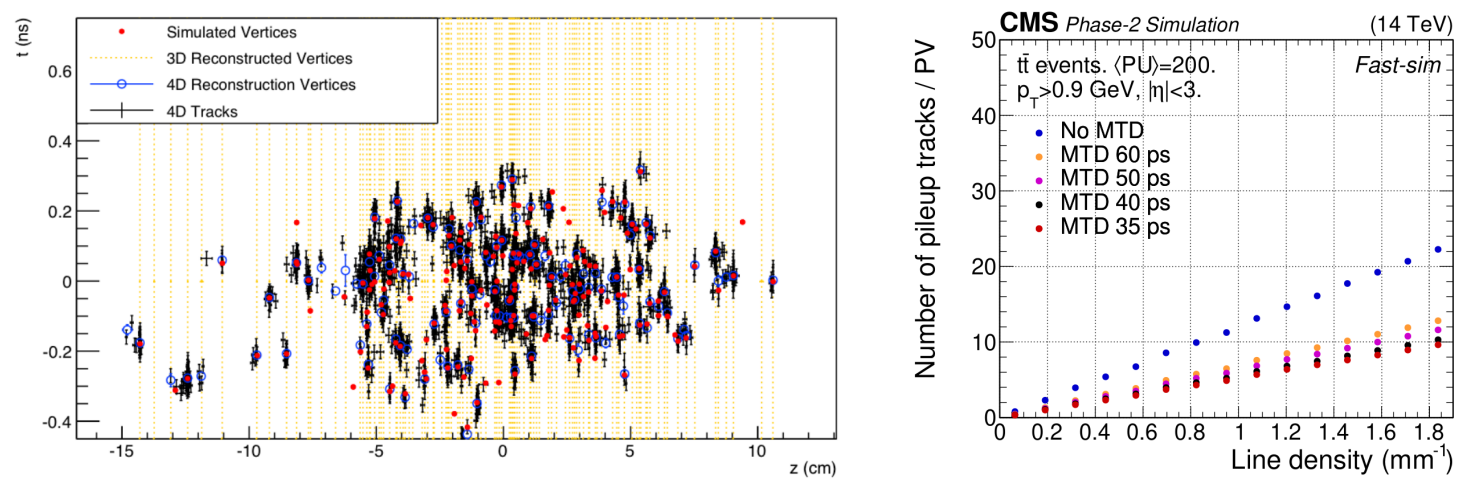

Figure 1: Left: Simulated and reconstructed vertices in a bunch crossing with 200 pileup interactions assuming a MIP timing detector with $\sim 30 \mathrm{ps}$ time resolution. The red dots are the simulated vertices, the vertical yellow lines indicate 3D-reconstructed (i.e. no use of timing information) vertices, while the black crosses and the blue open circles represent tracks and vertices reconstructed using a method that includes the time information (referred to as “4D”). Right: Number of pileup tracks incorrectly associated with the hard interaction vertex as a function of the collision line density for different time resolutions.

radius of $1188 \mathrm{~mm}$. The overall active length in $\mathrm{z}$ is of about $5000 \mathrm{~mm}$ yielding a total active surface of about $38 \mathrm{~m}^{2}$. The BTL will cover the pseudorapidity region up to $|\eta|=1.48$ and the fundamental detecting cell will consist of a thin LYSO:Ce crystal bar coupled at each end to a SiPM. Every LYSO:Ce crystall will be $5.7 \mathrm{~cm}$ long in the phi direction of CMS and $3.0 \mathrm{~mm}$ thick in the $\mathrm{z}$ direction. The radial thickness will vary from $3.7 \mathrm{~mm}$ for $|\eta|<0.7,3.0 \mathrm{~mm}$ for $0.7<|\eta|<1.1$ and $2.4 \mathrm{~mm}$ for $|\eta|>1.1$, in such a way the slant thickness crossed by the particles will be constant. The SiPMs will operate above the breakdown voltage in Geiger mode with a gain of the order of $10^{5}$. As the over-voltage (OV) produces a dark current that grows with the accumulated radiation dose, the SiPMs will be operated at low temperatures of about $-45^{\circ} \mathrm{C}$. The full detector will be cooled down using the CMS Tracker detector $\mathrm{CO}_{2}$ cooling system, while SiPMs will be locally cooled down by by another $10^{\circ} \mathrm{C}$ with thermo-electric coolers (TEC). The SiPM operation voltage will have to be smoothly decreased during the detector lifetime to limit the noise level while maintaining good time resolution. The electronics must also be designed to handle large leakage currents. In particular, a dedicated ASIC, named the TOFHIR (Time-of-flight, High Rate) chip, will be used to deliver precision timing information from the SiPM signals.

Regarding the MTD design geometry, as shown in Figure 2, the BTL will be made of 72 azimuthal segments (trays), 36 in each z-side, spanning $10^{\circ}$ in $\phi$. Each tray has a row of six Readout Units (RUs), every RU is made of twelve Front-End (FE) boards connected to a Concentrator Card (providing low voltage power, bias voltage, and three lpGBTs), and to every FE a module made of two LYSO:Ce arrays (16 crystals and 32 SiPMs each) is connected.

LYSO:Ce crystals were chosen because of the high density $\left(7.1 \mathrm{~g} / \mathrm{cm}^{3}\right.$, most probable value of energy deposit by a MIP $\sim 0.86 \mathrm{MeV} / \mathrm{mm}$ ), high light yield ( 40000 photons $/ \mathrm{MeV}$ ), fast scintillation rise time ( $<100 \mathrm{ps})$, short decay time ( $\sim 40 \mathrm{~ns})$ and very high radiation tolerance (less than few percent transmission loss at the end of HL-LHC). In addition, LYSO:Ce crystals have the scintillation light at $420 \mathrm{~nm}$ wavelength, matching perfectly with the SiPMs sensitivity. On the other hand, SiPMs were chosen because they are compact, robust, insensitive to magnetic field, and have a $20-40 \%$ 


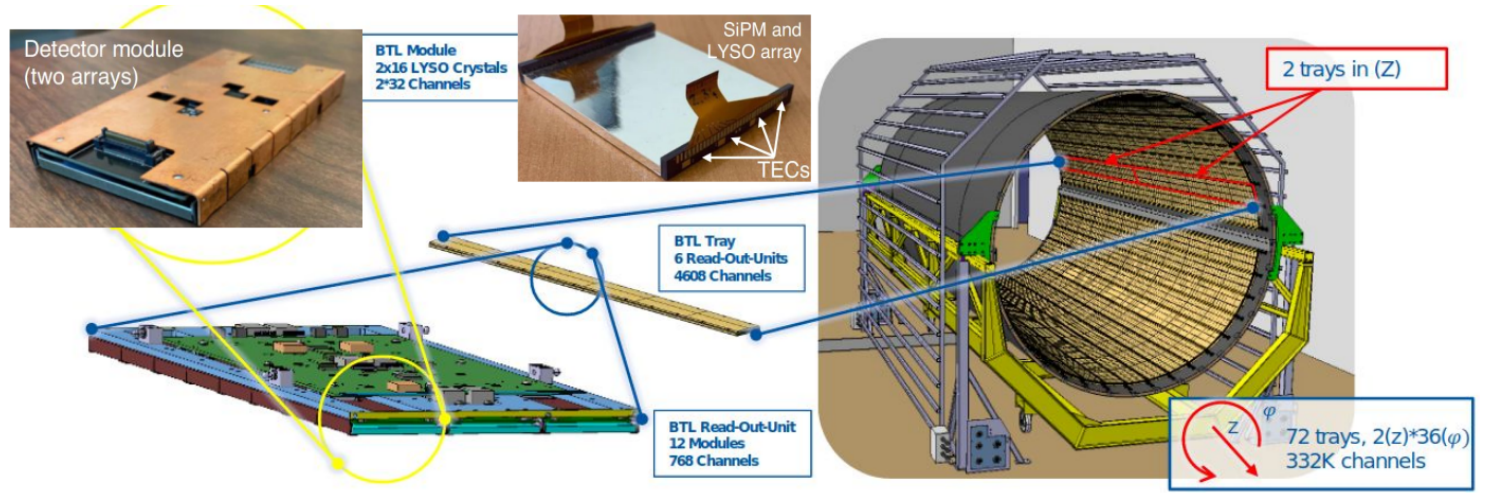

Figure 2: Overview of the BTL showing (left) the hierarchical arrangement of the various components, bars, modules, and Readout Units, and (right) trays inside the TST.

photo-detection efficiency (PDE) with an optimal cell size of $15 \mu \mathrm{m}$. The main challenge in using the SiPMs under HL-LHC radiation dose is the increase of the dark current rate and the self-heating of the sensors. In order to mitigate the impact on the performance of the timing resolution, a dedicated noise cancellation circuit is present inside the ASIC chip (TOFHIR), the annealing of the SiPMs to about $40^{\circ} \mathrm{C}$ during shutdown will be done, and thermo-electric coolers (TECs) will operate to reduce SiPMs temperature. Thanks to this, at the end of the HL-LHC phase a timing resolution of $\sim 50-60 \mathrm{ps}$ is expected.

\section{Test beam studies}

The tests were performed at the Fermilab Test Beam Facility (FTBF), using $120 \mathrm{GeV}$ protons, irradiating single LYSO:Ce bars read out by SiPMs prototypes. The experimental setup is shown in Figure 3. The trigger was based on a $10 \mathrm{~cm}^{2}$ scintillation counter located a few meters upstream with respect to the experimental setup. A silicon tracker telescope, consisting of twelve strip modules with $60 \mu \mathrm{m}$ pitch in alternating orientation, was located in front of the crystals and SiPMs under test to determine the impact point of the beam particles with a precision of about $0.2 \mathrm{~mm}$. The crystals and SiPMs were located inside a dark box with its temperature maintained at about $25 \pm 1^{\circ} \mathrm{C}$. The box was mounted on a support structure capable of rotating the sensors with respect to the beam direction. A Photek 240 Micro Channel Plate-PMT (MCP-PMT), with a time resolution of about $12 \mathrm{ps}$, was positioned along the beam line just downstream the box and was used to measure a reference time.

The crystal bars of LYSO:Ce used in this test were manufactured by Crystal Photonics Inc. (CPI) in three different geometries of $3 \times \mathrm{t} \times 57 \mathrm{~mm}^{3}$, where the thickness, $t$, varies between 2,3 and 4 $\mathrm{mm}$. Two different SiPM types were tested, the first type belongs to a set of S12572-015 SiPMs from Hamamatsu (HPK) with an active area of $3 \times 3 \mathrm{~mm}^{2}$, while the second set was provided by Fondazione Bruno Kessler (FBK), with an active area of $3 \times 3 \mathrm{~mm}^{2}$ based on the NUV-HD-ThinEpi technology. The HPK and FBK SiPMs provide gains of $1.8 \times 10^{5}$ and $2.5 \times 10^{5}$ respectively, and the PDE weighted by the emission spectra of LYSO:Ce is similar for the two SiPMs and reaches about $36 \%$ for $6 \mathrm{~V}$ over-voltage. Instead of the design readout electronics (ASIC boards on FE con- 


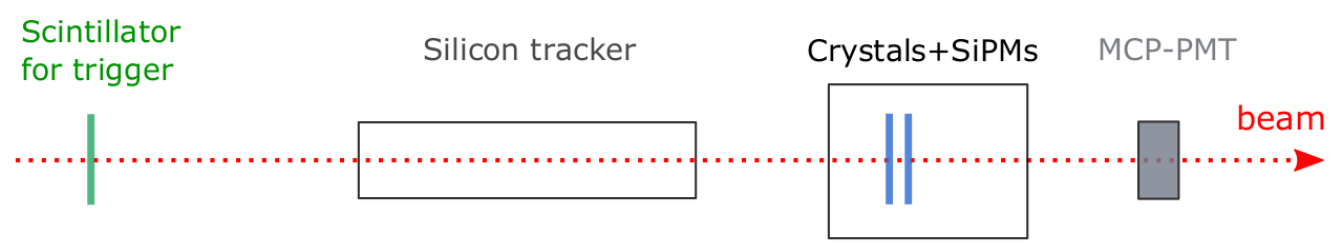

Figure 3: Schematic view of the beam line. From left to right, the scintillator is used for the trigger, the silicon tracker defines the MIP impinging position in the $x y$-plane, the MCP-PMT is used to define the reference time. The two crystal+SiPMs test setups are positioned inside a box, along the beamline.

nected to CCs), customized electronic boards were used to apply the bias and perform the readout of the SiPMs, and the full pulse shapes were digitized with a sampling frequency of about 5.12 GSample/s. The waveforms corresponding to the signals of each SiPM were analysed to extract the signal amplitude and the time at which the MIP crossed the sensor. In particular, the subtraction of the pedestal is performed and the timing information is extracted using a leading edge discrimination.

\section{Results}

The final performance of the timing reconstruction is studied for different references, after amplitude walk corrections and position corrections are applied. In particular, amplitude walk corrections are calculated measuring the dependence of $t_{S i P M}-t_{M C P}$ on the amplitude of the SiPM signal, while position corrections are computed measuring the dependence of $t_{\text {left }}+t_{\text {right }}$ on the impinging position of the MIP on the bar. Position uniformity of the timing resolution is measured for the following references for different SiPM manufactures (HPK and FBK):

- $\Delta t_{\text {average }}=\frac{1}{2}\left(t_{\text {left }}+t_{\text {right }}\right)-t_{M C P}$

- $\Delta t_{\text {diff }}=t_{\text {left }}-t_{\text {right }}$

- $\Delta t_{\text {left }}=t_{\text {left }}-t_{M C P}$

- $\Delta t_{\text {right }}=t_{\text {right }}-t_{M C P}$

As shown in Figure 4 (top) a very stable spatial uniformed can be reached throughout the full crystal for both SiPM manufactures and different crystal dimensions. In addition, a global and local resolution is computed, Figure 4 (down). In all the measurements the target time resolution is achieved, demonstrating the feasibility of the BTL design. Additional studies to demonstrate that this resolution is retained also with the usage of the full electronics system (TOFHIR ASIC chip on FE connected to $\mathrm{CC}$ unit) are ongoing, and will demonstrate the feasibility of the full chain design of the BTL. 

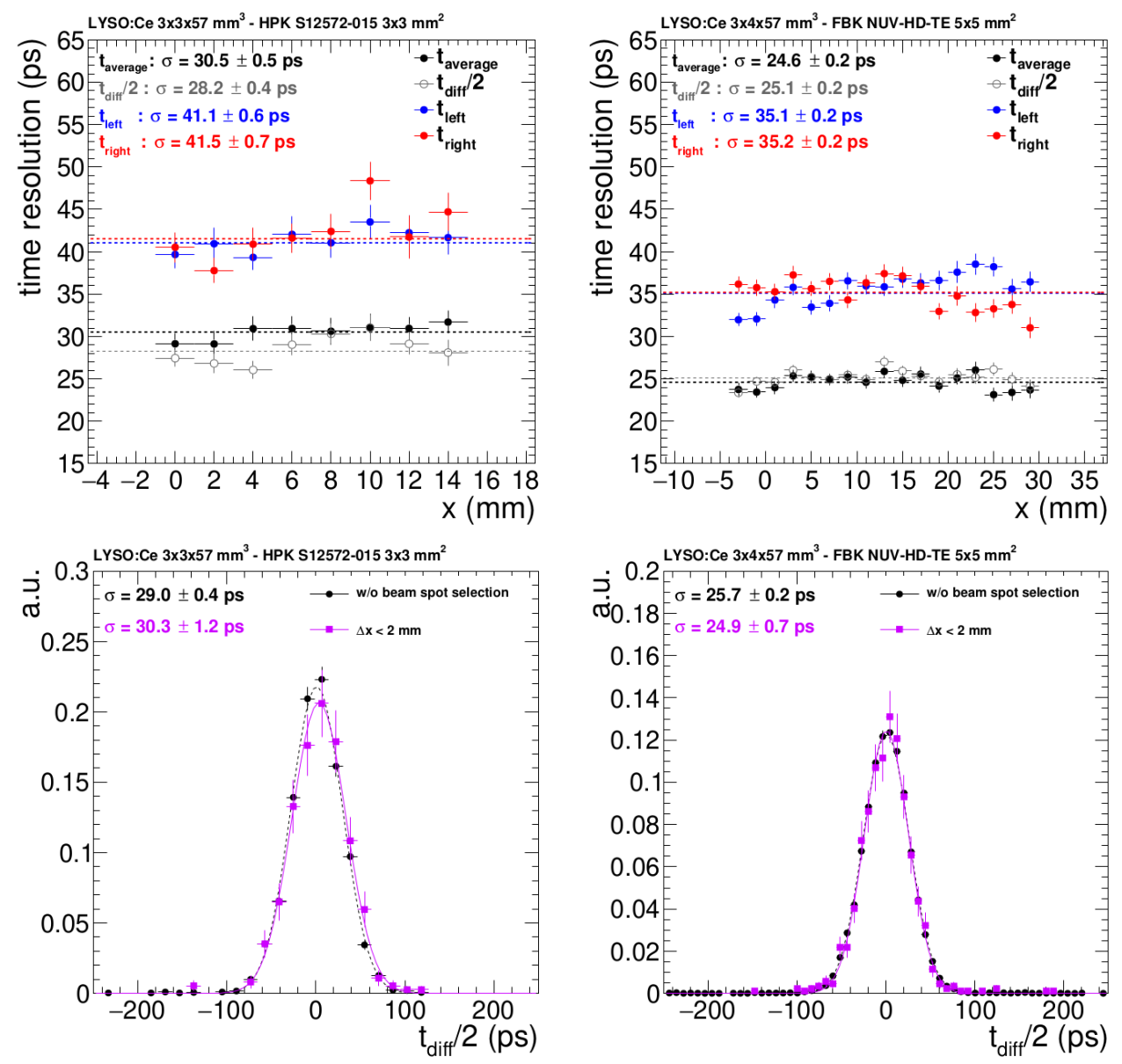

Figure 4: Top: Time resolution of the left and right SiPMs, their average, and half of the time difference as a function of the MIP impact point for a $3 \times 3 \times 57 \mathrm{~mm}^{3}$ LYSO:Ce bar coupled to HPK SiPMs (left) and for a $3 \times 4 \times 57 \mathrm{~mm}^{3} \mathrm{LYSO}: \mathrm{Ce}$ bar coupled to FBK SiPMs (right).. Bottom: global and local time resolution for a $3 \times 3 \times 57 \mathrm{~mm}^{3}$ LYSO:Ce bar coupled to HPK SiPMs (left) and for a $3 \times 4 \times 57 \mathrm{~mm}^{3}$ LYSO:Ce bar coupled to FBK SiPMs (right).

\section{References}

[1] CMS collaboration, "The CMS experiment at the CERN LHC", JINST 3 S08004 (2008) DOI:10.1088/1748-0221/3/08/S08004

[2] CMS collaboration, "A MIP Timing Detector for the CMS Phase-2 Upgrade", CMS-TDR-020 cds:2667167

[3] CMS MTD collaboration, "Test beam characterization of sensor prototypes for the CMS Barrel MIP Timing Detector", JINST 16 (2021) DOI: 10.1088/1748-0221/16/07/P07023, arxiv:2104.07786 